\title{
High Temperature Unimolecular Decomposition of Cyclopentanone
}

Binod Raj Giri ${ }^{1}$, Mohammed AlAbbad ${ }^{1}$, John R. Barker ${ }^{2, *}$ and Aamir Farooq, ${ }^{1, *}$

${ }^{1}$ King Abdullah University of Science and Technology (KAUST), Clean Combustion Research Center, Physical Sciences and Engineering Division, Thuwal, Saudi Arabia

${ }^{2}$ Department of Climate and Space Sciences \& Engineering, University of Michigan, Ann Arbor, Michigan 48109-2143, United States

*Corresponding Authors: Aamir.Farooq@kaust.edu.sa; jrbarker@umich.edu

\section{Colloquium: Gas-Phase Reaction Kinetics}

Word Count (method1):

\begin{tabular}{|l|l|}
\hline Main text & 3581 \\
\hline Equations: 5 & 76 \\
\hline References: 33 & 611.8 \\
\hline Figures: 4 & 823.2 \\
\hline Tables: 0 & 0 \\
\hline Total & $\mathbf{5 0 9 2}$ \\
\hline
\end{tabular}

This paper includes Supplementary Material. 


\section{Abstract}

2 This work reports thermal decomposition of cyclopentanone behind reflected shock waves over 1150

$3-1590 \mathrm{~K}$ and $750-1800$ Torr. Carbon monoxide is one of the main reaction products and its formation

4 was monitored using a quantum cascade laser operating near $4.56 \mu \mathrm{m}$. Our results show that

5 cyclopentanone undergoes decomposition, under the present experimental conditions, via reaction

6 channels that produce $\mathrm{CO}$ almost exclusively. A recent $a b$ initio study by Zaras et al. (Int J Chem Kinet

$747,2015,439-446)$ revealed that cyclopentanone decomposes to produce CO and ethylene by two

8 channels: ring-opening to form a di-radical which subsequently decomposes, and concerted

9 elimination to produce $\mathrm{CO}$ and $\mathrm{C}_{2} \mathrm{H}_{4}$ directly; their predicted rate constants are much slower than

10 literature experimental data. To resolve the rate constant discrepancy and to determine whether keto-

11 enol tautomerism plays a significant role, we performed master equation simulations which produced

12 results in good agreement both with the previous $a b$ initio study and with the experimental data

13 obtained in the present work.

16 Keywords: Cyclopentanone; Unimolecular decomposition; Ab initio; Carbon monoxide; Laser absorption. 
18 Ketones are of significant interest as potential biofuels. Several researchers have demonstrated the

19 use of chemical and biochemical conversion process technology for biofuel production [1]. One such

20 technology involves using endophytic fungi to decompose lignocellulosic biomass, agro-industrial

21 residues, and wastewater to produce a wide array of volatile organic compounds (VOCs), including

22 saturated and unsaturated hydrocarbons, ketones, esters, acids, and alcohols [1-4]. These oxygenated

23 compounds can promote cleaner combustion by reducing emissions of greenhouse gases and

24 particulates. Ketones are also important intermediates in the low-temperature oxidation of large

25 hydrocarbons and oxygenated fuels such as alcohols and esters. Therefore, the reaction kinetics of

26 ketones is of substantial interest.

27 Cyclopentanone (CPO) is a five-member ring cyclic ketone that can be efficiently produced from 28 hydrogenation of biomass derived furfural [5], and the pyrolysis of biomass [6] and of nylon 66 [7]. It 29 is highly resistant to auto-ignition and can potentially be utilized in fuel blends for spark-ignition 30 internal combustion engines. It may also prove to be a promising feedstock for synthesis of polycyclic 31 alkanes [8,9] which are known as high-density fuels [10], and it may be used as aviation fuel as well 32 as in blends with increased volumetric heating values. Despite its importance in combustion, the 33 kinetics of CPO has received very little attention [11-17].

34 Cyclopentanone decomposition has been studied experimentally by using photochemical [11,12] and 35 thermal $[13,14]$ methods, and computationally by ab initio/master equation methods [15]. The first 36 study dates back to 1935, when Saltmarsh and Norrish[11] carried out photochemical decomposition 37 of cyclic ketones with $n$-membered rings that contained 5 to 7 carbon atoms $(5 \leq n \leq 7)$. They concluded 38 that these cyclic ketones primarily decompose via simultaneous rupture of both $\mathrm{C}-\mathrm{C}$ bonds adjacent to 39 the carbonyl group, forming $\mathrm{CO}$ and cyclic alkanes $(n-1)$ as final products. However, in the case of $40 \mathrm{CPO}$, they identified ethylene, butene isomers $\left(\mathrm{C}_{4} \mathrm{H}_{8}\right)$ and $\mathrm{CO}$ as the decomposition products. They 41 hypothesized that the decomposition of cyclic ketones can produce cyclic hydrocarbons when there is 
42 a possibility for the formation of a 5 or 6 membered ring. In a later photolysis study, Benson and 43 Kistiakowsky [12] suggested that the $\mathrm{C}_{4} \mathrm{H}_{8}$ identified by Saltmarsh and Norrish [11] as butene isomers 44 is actually cyclobutane $\left(\mathrm{C}_{4} \mathrm{H}_{8}\right)$. They questioned the experimental techniques employed by Saltmarsh 45 and Norrish [11] and further stated that the photolysis of cyclic ketones is much more complex than 46 the simple hypothesis advanced by Saltmarsh and Norrish [11]. Somewhat later, Johnson and Walters

47 [13] studied the thermal decomposition of CPO in a cylindrical pyrex reaction vessel at $761-816 \mathrm{~K}$ 48 and 99 - 314 Torr, and reported that the major products in the early stages of reaction are $\mathrm{CO}, \mathrm{C}_{2} \mathrm{H}_{4}$, $49 \mathrm{H}_{2}$, 1-butene, 2-cyclopenten-1-one. They found that the reaction is not elementary since it exhibited 50 an induction period and was autocatalytic, i.e., it was accelerated by the addition of the reaction 51 products. In the most recent experimental study of CPO, Delles et al. [14] conducted experiments at $52805-854 \mathrm{~K}$ and $11-30$ Torr, and observed major products similar to those reported by Johnson and 53 Walters [13] but they additionally identified 4-pentenal as an important product, and reported that the 54 over-all thermal decomposition of CPO can be described by $k(T)=1.5 \times 10^{12} \exp (-29642.7 / T) \mathrm{s}^{-1}$. 55 Delles et al. [14] confirmed that the reaction is autocatalytic and also that its rate is reduced by the 56 addition of nitric oxide, suggesting participation by a free radical chain mechanism under their conditions. All of these experimental studies were confined to temperatures lower than $855 \mathrm{~K}$ and the most recent study [14] was performed almost 50 years ago.

Recently, Zaras et al. [15] employed G3B3/master equation-RRKM computational methods to 60 investigate the various decomposition pathways for CPO decomposition. The authors [15] were able 61 to map out seven different reaction routes that led to the major decomposition products observed 62 previously in the pyrolysis experiments. Their calculations showed that the reaction route leading 63 directly to $\mathrm{C}_{2} \mathrm{H}_{4}$ and $\mathrm{CO}$ is the dominant channel and is significantly faster than keto-enol tautomerism, 64 particularly at high temperatures. The authors further reported that enolization is less favored in CPO 65 than in cyclohexanone, since the latter has a lower barrier for the corresponding reaction. Their 66 calculated rate coefficients for CPO decomposition are at least an order of magnitude slower than the 
67 experimental rate expression reported by Delles et al. [14].

68 The present work was motivated by the discrepancy between theory and experiment and by the

69 problems encountered in the early experimental works which employed classical macroscopic kinetics 70 techniques. Experimental techniques have advanced greatly since those days. The objectives behind 71 the present work are several-fold: (i) obtain experimental rate constants for the thermal decomposition 72 of $\mathrm{CPO}$ at high temperatures which are more relevant for combustion systems, (ii) perform statistical 73 rate theory calculations for comparison to the experiments and attempt to resolve the discrepancy 74 between theory and experiment, (iii) assess of the role of the keto-enol tautomerization channel.

2. Experimental Setup

Thermal unimolecular decomposition of cyclopentanone (CPO) was investigated in the low-pressure shock tube (LPST) facility at King Abdullah University of Science and Technology (KAUST). As the details of LPST can be found elsewhere [18], we provide here only a brief description. The LPST, fabricated from stainless steel, has driver and driven sections with an inner diameter of $14.2 \mathrm{~cm}$. The 80 length of the driver section can be varied to achieve the desired reaction time behind reflected shock 81 waves. This work used a 3 meter long driver section to achieve test times of about $1.5 \mathrm{~ms}$. The driven 82 section was separated from the driver section by a polycarbonate diaphragm. To ensure high purity 83 prior to each experiment, the driven section was pumped down to $<10^{-5}$ Torr using a turbo-molecular 84 pump and the driver section was evacuated to $10^{-3}$ Torr. Thereafter, the driven and driver sections were 85 filled with the cyclopentanone-argon mixture and helium gas, respectively. Shock waves were 86 generated by gradually increasing the helium pressure in the driver section until the diaphragm 87 ruptured. The incident shock wave speed was measured by a series of five PCB 113B26 piezoelectric 88 pressure transducers (PZTs) placed in the last $1.3 \mathrm{~m}$ of the driven section. All experiments were 89 conducted behind reflected shock waves, and the post-shock conditions $\left(T_{5}, P_{5}\right)$ were calculated, with 
90 a maximum uncertainty of $1 \%$, using standard shock-jump relations [19] embedded in the Frosh code.

91 [20]. Optical windows for the CO laser diagnostic and a Kistler PZT were located at $2 \mathrm{~cm}$ from the 92 shock tube endwall.

93 Carbon monoxide was monitored using the $\mathrm{R}(13)$ absorption line near $2193.36 \mathrm{~cm}^{-1}(4.56 \mu \mathrm{m})$ in

94 the $v_{1}$ fundamental vibrational band of CO. A fixed-wavelength direct absorption strategy was used,

95 where the mid-IR light was generated by a cw quantum cascade laser (QCL) supplied by Alpes Lasers.

96 The mole fraction of CO was obtained using the Beer-Lambert law: $I / I_{0}=\exp \left(-S \phi x_{i} P_{5} L\right)$ where $I$ and

$97 I_{0}$ are the transmitted and incident laser intensities, respectively; $S\left(\mathrm{~cm}^{-2} \mathrm{~atm}^{-1}\right)$ is the line-strength of 98 the ro-vibrational absorption transition, $\phi(\mathrm{cm})$ is the line-shape function, $x_{i}$ is the mole fraction of the 99 absorbing species, $P_{5}(\mathrm{~atm})$ is the total pressure of the gas behind the reflected shock wave and $L(\mathrm{~cm})$ 100 is the absorption path length (internal diameter of the shock tube). Argon-broadening parameters for 101 the $R(13)$ transition were taken from Ren et al.[21] and the line-strength was obtained from the 102 HITRAN database [22].

104 Gas mixtures of cyclopentanone ( $\geq 99 \%$ Sigma-Aldrich; further purified through freeze-pump-thaw 105 cycles), diluted in argon (99.999\%) were prepared manometrically in a $24 L$ teflon-coated stainless 106 steel vessel equipped with a magnetically driven stirrer. Prior to mixture preparation, the vessel was 107 pumped down to $<10^{-5}$ Torr. Mixtures were allowed to homogenize for at least two hours before use, 108 and contained $0.2-1.2 \%$ CPO in argon. 


\section{3. Results and Discussion}

\subsection{Cyclopentanone Decomposition Pathways}

115 The major reaction pathways for the unimolecular decomposition of cyclopentanone are shown in

116 Fig. 1. The energetics for these pathways are taken from Zaras et al. [15] who used the G3B3 method

117 to compute the stationary points on the potential energy surface (PES).

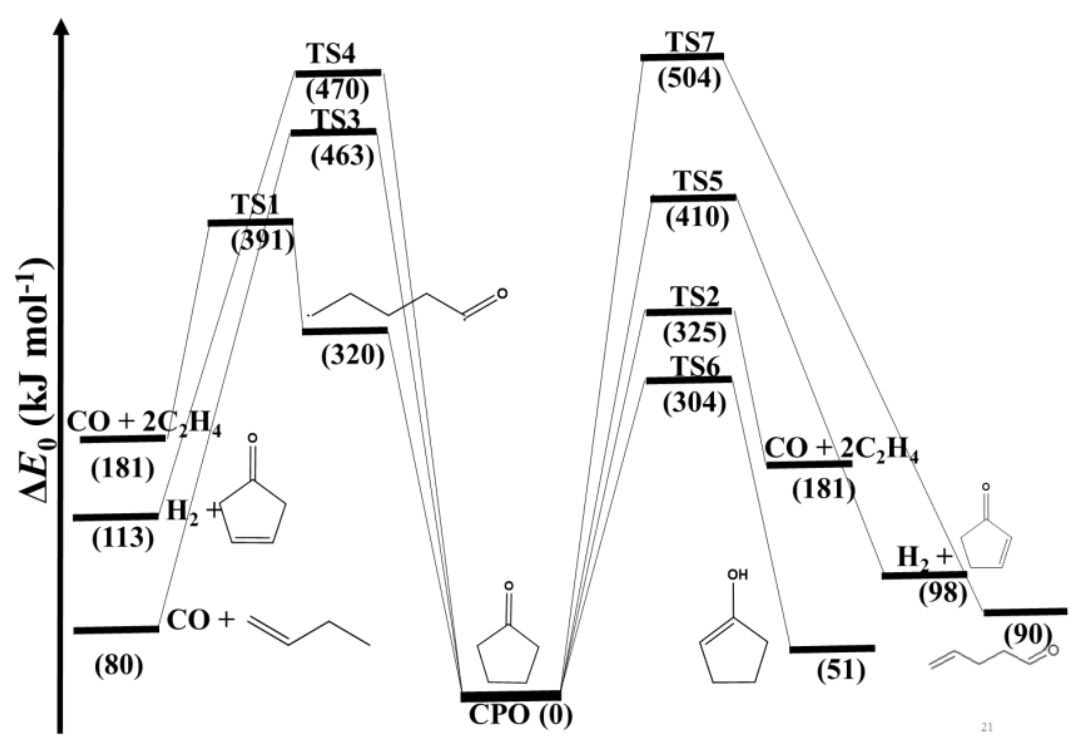

119 Fig. 1. G3B3 energies [15] for the major decomposition pathways of cyclopentanone (CPO).

120 As shown in Fig. 1, the energy barriers for the reaction pathways via TS3, TS4, TS5, and TS7 are 121 significantly higher than those proceeding via TS1, TS2 and TS6. Zaras et al. [15] predicted that the 122 higher energy channels make negligible contributions to the total decomposition rate. This suggests 123 that the higher energy channels can safely be neglected in the analysis of our kinetic data, leaving only 124 the following three reactions to be considered: 
128 Two distinct reaction pathways, R1 and R2, which occur via TS1 and TS2, respectively, lead to the 129 same set of products: $\mathrm{CO}$ and $\mathrm{C}_{2} \mathrm{H}_{4}$. Reaction pathway $\mathrm{R} 1$, which is indirect, first surmounts a 130 thermodynamic barrier of $326.8 \mathrm{~kJ} / \mathrm{mol}$ (reaction R1a has no intrinsic barrier) to open the ring and 131 produce an open chain diradical $\left({ }^{\circ} \mathrm{CH}_{2}\left(\mathrm{CH}_{2}\right)_{3} \mathrm{C}^{\circ}=\mathrm{O}\right)$, which reacts further by a low energy path 132 (reaction $\mathrm{R} 1 \mathrm{~b}$ with an energy barrier of $64.4 \mathrm{~kJ} / \mathrm{mol}$ relative to the diradical) to produce $\mathrm{CO}$ and $\mathrm{C}_{2} \mathrm{H}_{4}$. 133 As shown in Fig. 4 of Zaras et al. [15], the open chain diradical can also isomerize to 4-pentenal by 134 overcoming an energy barrier of $301.3 \mathrm{~kJ} / \mathrm{mol}$ (reaction R1c - not shown in Fig. 1). However, reaction $135 \mathrm{R} 1 \mathrm{c}$ is not important, because its barrier height is so much greater than that of reaction R1b.

$136{ }^{\circ} \mathrm{CH}_{2}\left(\mathrm{CH}_{2}\right)_{3} \mathrm{C}^{\bullet}=\mathrm{O} \rightarrow \mathrm{CO}+2 \mathrm{C}_{2} \mathrm{H}_{4}$

$137{ }^{\circ} \mathrm{CH}_{2}\left(\mathrm{CH}_{2}\right)_{3} \mathrm{C}^{\bullet}=\mathrm{O} \rightarrow \mathrm{CH}_{2}=\mathrm{CHCH}_{2} \mathrm{CH}_{2} \mathrm{C}(\mathrm{H})=\mathrm{O}$

138 Reaction $\mathrm{R} 2$, the second pathway that produces $\mathrm{CO}$ and $\mathrm{C}_{2} \mathrm{H}_{4}$, proceeds directly in a concerted manner 139 via TS2.

140 A third pathway, reaction R3, proceeds first by an enolization step to produce cyclopentenol (keto141 enol tautomerization via TS6 with energy barrier $304 \mathrm{~kJ} / \mathrm{mol}$ ), which reacts further to produce various 142 products, as shown in Figure 4 of Zaras et al. [15]. The enolization step via TS6 has a higher energy 143 barrier than the analogous process in cyclohexanone (276 kJ/mol [23]), but it is lower in energy than 144 pathways R1 and R2. Although it is lower in energy, the CPO enolization reaction on pathway R3 has 145 a very tight transition state $\left(\Delta S_{298.15 \mathrm{~K}^{\neq}}=-3.3 \mathrm{~J} \mathrm{~mol}^{-1} \mathrm{~K}^{-1}\right)$, while the transition states along pathways $146 \mathrm{R} 1 \mathrm{a}$ and R2 are much looser $\left(\Delta S_{298.15 \mathrm{~K}}{ }^{\neq}=18-38.5\right.$ and $40.2 \mathrm{~J} \mathrm{~mol}^{-1} \mathrm{~K}^{-1}$, respectively). The net result 147 is that the channels producing $\mathrm{CO}$ and $\mathrm{C}_{2} \mathrm{H}_{4}$ are more important than channel $\mathrm{R} 3$.

148 For the analysis of experimental data, a further simplification is possible (and supported by the 149 theoretical calculations described below). Because reactions R1b and R1c occur via tight transition 150 states with significant energy barriers, while the reverse of reaction R1a (recyclization of the open 
151 chain diradical) is barrierless and hence very rapid, subsequent reaction to form $\mathrm{CO}+\mathrm{C}_{2} \mathrm{H}_{4}$ cannot 152 compete with R2. Therefore, the indirect channel forming $\mathrm{CO}$ and $\mathrm{C}_{2} \mathrm{H}_{4}$ can be neglected in analyzing 153 the experimental data on CPO decomposition.

\section{$154 \quad 3.2 \quad$ Experimental Rate Coefficients}

155 From the discussion above, the most favored decomposition channels of CPO decomposition are 156 expected to be the reactions $\mathrm{R} 2$ and $\mathrm{R} 3$. $\mathrm{R} 2$ forms $\mathrm{CO}$ and $\mathrm{C}_{2} \mathrm{H}_{4}$ directly, while $\mathrm{R} 3$ is the enolization 157 channel which eventually leads to the formation of other products. Because CO is one of the major 158 products, we monitored the evolution of $\mathrm{CO}$ to extract the kinetic information. Our results show that 159 the $\mathrm{CO}$ yield approaches unity $\left([\mathrm{CO}]_{\infty} /[\mathrm{CPO}]_{0} \approx 1\right)$ at high temperatures and/or long reaction times 160 (see Fig. 2). Moreover, the measured evolution of CO starts at the onset of reaction at high temperatures 161 which clearly indicates that R2 is the major decomposition pathway. This observation is consistent 162 with the theoretical predictions of Zaras et al. [15].

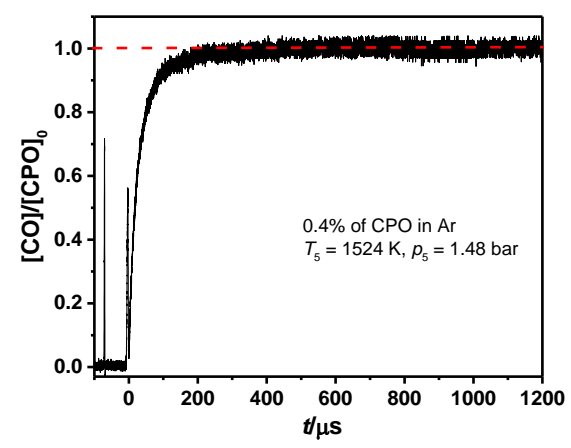

164 Fig. 2. Measured [CO $] /[\mathrm{CPO}]_{0}$ time history for the thermal unimolecular decomposition of cyclopentanone 165 (CPO). The red line shows that $\mathrm{CO}$ yield reaches unity at high temperatures and/or long reaction times.

166 The reaction rate coefficients $k(T, p)$ were extracted by iteratively varying the rate coefficients for 167 R2 and R3 until the best fit of the measured absorbance time-profiles of CO was obtained. ChemkinPro 168 was used for the kinetic simulation using the detailed cyclopentanone oxidation mechanism from 169 Thion et al. [16], and the initial estimates of the rate coefficients for R2 and R3 were taken from Zaras 
170 et al. [15]. The best-fit kinetic simulations are shown in Fig. 3 and the total rate coefficients for the 171 loss of CPO in each experiment are plotted in Fig. 4 (and compiled in Table S1 of the Supporting 172 Information). The measured rate coefficients, obtained at pressures ranging from 750 to 1800 Torr, did 173 not exhibit any discernible pressure dependence. This suggests that the CPO decomposition is close to 174 the high-pressure limit under the present experimental conditions.

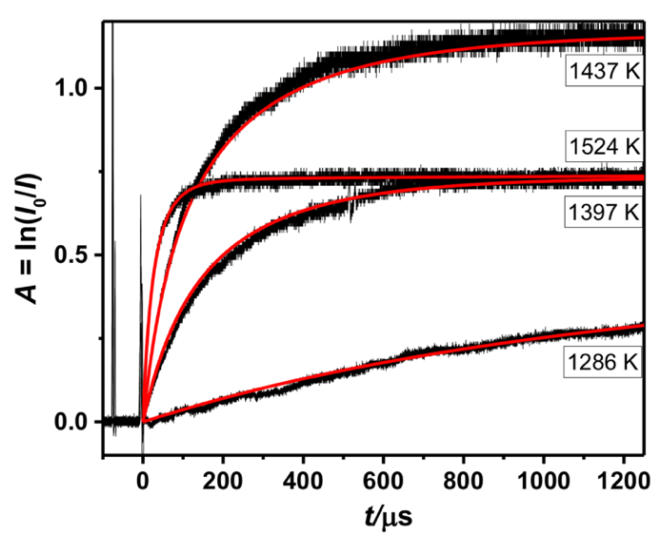

176 Fig. 3. Representative absorbance time-profiles for the formation of $\mathrm{CO}$ from the thermal decomposition of 177 cyclopentanone. Black lines are the experimental traces and red lines indicate the best fit of kinetic simulations 178 at the specified temperatures. Further details of the experimental conditions are provided in Table S1 of the 179 Supporting Information.

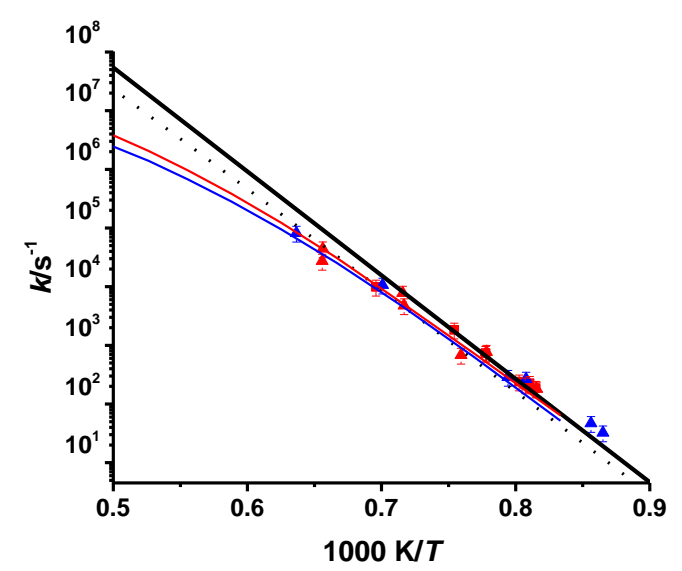

181 Fig. 4. Arrhenius plot for the experimental and theoretical rate coefficients for the thermal decomposition of

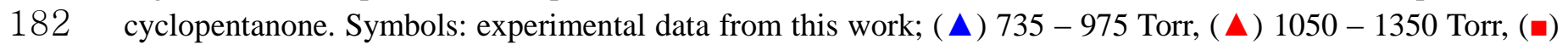
$1831425-1800$ Torr. Solid lines: results of our master equation calculations for $p=\infty, 1500$ and 750 Torr; ( ....) 184 Zaras et al. [15] values for the total high-pressure limiting rate coefficients. The error bars $( \pm 25 \%)$ of our 185 measured rate constants account uncertainties in the temperature $( \pm 1 \%)$ of reflected shock wave, location of 186 time zero $( \pm 0.5 \mu \mathrm{s})$, mixture composition $( \pm 5 \%)$, CO absorption cross section $(\sim 3 \%)$. 
188 Our experimental data compare reasonably well with the theoretical predictions of Zaras et al. [15] 189 (dashed line in Fig. 4), except for the difference in temperature-dependence and, at the lower 190 temperatures, the experimental rate constants are at least twice as fast as the $k_{\infty}$ predicted by Zaras et 191 al. [15]. This suggests that the energy barriers predicted by the theory for the low-lying channels are 192 somewhat too high. For that reason, we empirically reduced the energy barrier of TS2 (direct pathway 193 to $\left.\mathrm{CO}+\mathrm{C}_{2} \mathrm{H}_{4}\right)$ by $6.3 \mathrm{~kJ} / \mathrm{mol}(1.5 \mathrm{kcal} / \mathrm{mol})$, which is within the range of accuracy expected for this 194 level of theory. After making this adjustment, the computed pressure- and temperature- dependent rate 195 coefficients (solid lines in Fig. 4) and branching ratios are in good agreement with the experimental 196 data as described in the next section.

\section{$197 \quad 3.3$ Master Equation Simulations}

198 Pressure- and temperature- dependent rate coefficients were computed by using the MultiWell 199 Master Equation Program Suite [24-26]. MultiWell uses stochastic simulations to solve the 1-D master 200 equation which depends on total internal energy, as outlined by Smith and Gilbert [27] and slightly 201 extended by others [28]. Full descriptions of the code and underlying theory can be found in the 202 literature citations and the User Manual.

203 In these calculations, argon was used as bath gas and the energy transfer was treated using the 204 exponential-down model with $\langle\Delta E\rangle_{\mathrm{d}}=200(T / 300 \mathrm{~K})^{0.85} \mathrm{~cm}^{-1}[15,23,29]$. The bimolecular rate 205 coefficients describing the collisions between argon and the reactant molecule or the intermediate wells 206 are based on Lennard-Jones (LJ) collisions parameters, which were obtained from the parameters of 207 the individual collision partners by using the standard combination rules. The LJ parameters used were $208 \sigma=3.47 \AA$ and $\varepsilon / \mathrm{k}_{\mathrm{b}}=114 \mathrm{~K}$ for $\mathrm{Ar}$ [30], and $\sigma=5.5 \AA$ and $\varepsilon / \mathrm{k}_{\mathrm{b}}=650.7 \mathrm{~K}$ for CPO [15]. For the 209 molecular properties, we used the G3B3 parameters reported by Zaras et al. [15] for reactions R1, R2 210 and R3 except for the missing parameters for the step cyclopentenol $\rightarrow$ cyclopentadienol $+\mathrm{H}_{2}$ of the 211 reaction sequence R3. For this step, we performed additional G3B3 calculations (with the same basis 
212 set used by Zaras et al. [15]) to obtain the energetics and transition state molecular parameters. The 213 calculated value of the barrier height for this step is found to be $251 \mathrm{~kJ} / \mathrm{mol}$, in exact agreement with 214 Zaras et al. [15]. The molecular parameters and the geometries are listed in Table S2 and S3 of the 215 Supporting Information.

216 Most of the rate constants in the master equation calculations were calculated from the molecular 217 parameters by using the RRKM theory, i.e., microcanonical transition state theory (TST). Angular 218 momentum-resolved microcanonical variational TST (i.e., VTST) theory was used to compute rate 219 constants for the barrierless reaction R1a. For that purpose, the molecular parameters were taken from 220 Zaras et al. [15], who performed relaxed scans at fixed points along the reaction path between 2.1 and $2213.5 \AA$ at intervals of $0.1 \AA$ (defined by the fissioning $\mathrm{C}-\mathrm{C}$ bond distance). These parameters were used 222 by the code MultiWell/Ktools to compute the microcanonical VTST rate constants for reaction path 223 R1a (the Ktools input data are reported in Supporting Information). The rotational and vibrational 224 parameters of the transition state for reaction R1b were not reported by Zaras et al. [15]. Since this 225 reaction is expected to be a minor pathway, the microcanonical rate constants needed for the 226 calculations were computed using the Inverse Laplace Transform (ILT) method [31], based on the 227 critical energy reported by Zaras et al. [15] and an assumed Arrhenius $A$-factor, $A_{\infty}=10^{14} \mathrm{~s}^{-1}$, which is 228 appropriate for a tight transition state at the temperatures of interest [32]. Tests performed by using the 229 methods outlined below showed that the calculated values for $k_{\text {uni }}$ increased by only $\sim 10 \%$ when this $230 A$-factor was increased by a factor of 100.

231 At each temperature and pressure, master equation simulations were performed for simulated time 232 periods corresponding to $10^{4}$ collisions (the MultiWell input data are reported in Supporting 233 Information). In each case, the number of stochastic trials was set to $10^{5}$ or $10^{6}$, in order to reduce 234 stochastic noise to the level of $\leq 3 \%$ (1 standard deviation). After the initial transient period $(\leq 500$ 235 collisions in this case, based on stabilization of the average vibrational energy [33]) the simulated 236 decays of CPO were very nearly pure exponential functions and unimolecular rate constants were 
237 evaluated by assuming a pure exponential decay: $k_{\text {uni }}=\ln \left(f_{2} / f_{1}\right) /\left(t_{2}-t_{1}\right)$, where $f_{\mathrm{i}}$ is the fraction of CPO 238 at time $t_{\mathrm{i}} ; t_{1}$ and $t_{2}$ corresponded to $10^{3}$ and $10^{4}$ collisions, respectively.

239 The results of our calculations are summarized in Fig. 4 and in Tables S4 - S6 (Supporting 240 Information) which report values of $k_{\text {uni }}$, the stochastic uncertainties, and branching ratios for the 241 various pathways, respectively. As can be seen in Fig. 4, our calculated values of the high-pressure 242 limiting rate coefficients, $k_{\infty}(T)$, agree well with the experimental data, thus supporting the conclusion 243 that our experimental data are close to the high-pressure limit, perhaps somewhat below it. Most of 244 our experiments were carried out at pressures ranging from 1 to 2 bar, where pressure falloff makes a 245 contribution. The pressure falloff observed in the experiments at higher temperatures is captured well 246 by our master equation calculations, as shown in Fig. 4. Our calculations also predict that the 247 decomposition of $\mathrm{CPO}$ leads almost exclusively to $\mathrm{CO}$ and $\mathrm{C}_{2} \mathrm{H}_{4}(\geq 95 \%)$ via reaction $\mathrm{R} 2$ under our 248 experimental conditions, and that the reaction R1 is negligible. The lowest energy pathway (R3) 249 contributes $\lesssim 3 \%$ to the total product yield.

250 The calculations described above were performed after making a single empirical adjustment to the 251 ab initio quantum chemistry results i.e lowering the energy of TS2 by $6.3 \mathrm{~kJ} / \mathrm{mol}(1.5 \mathrm{kcal} / \mathrm{mol})$ below 252 the G3B3 value. We performed additional tests (at $1500 \mathrm{~K}$ and 1.5 bar) to determine whether adjusting 253 the energy of TS6 (without adjustments to any of the other ab initio energies) could achieve similar 254 good agreement with the experimental data. We found that when the energy of TS6 is lowered by 30 $255 \mathrm{~kJ} / \mathrm{mol}$ (a much greater adjustment than the expected errors in the quantum chemistry calculations), 256 the predicted rate constant is still $>15 \%$ too low, and the branching ratio for reaction R2 is only $71 \%$, 257 which is much smaller than the experimental value. It might be possible to match the experimental 258 value for the unimolecular rate constant by making larger adjustments to the barrier height of TS6, but 259 then the predicted branching ratio would be even smaller. We conclude that the semi-empirical model 260 utilizing the adjusted barrier for TS2 provides the best description of the reaction system that is 
261 currently available.

263 The calculated sum of the high-pressure limiting rate coefficients for all channels can be expressed 264 as $k_{\infty}(T)=3.2 \times 10^{16} \exp \left(-336 \mathrm{~kJ} \mathrm{~mol}^{-1} / \mathrm{R} T\right) \mathrm{s}^{-1}$ for temperatures in the range $800-2000 \mathrm{~K}$. This total 265 high-pressure rate constant and the extrapolated values of the experimental rate constants measured in 266 the present work are lower than the results reported by Delles et al. [14], despite the fact that their 267 experiments were performed at much lower pressures and, therefore, pressure fall-off should have been 268 more important. Their results (and those of Johnson and Walters [13]) showed clear evidence of an 269 induction period, autocatalysis, and quenching by nitric oxide, which suggested to them that the 270 reaction was proceeding at least in part by a free radical chain. The present results are consistent with 271 their interpretation and we conclude that the present results are a more accurate description of the 272 unimolecular reactions of cyclopentanone.

\section{Conclusions}

275 The kinetics of the thermal decomposition of cyclopentanone was studied behind reflected shock 276 waves at 1150 - $1590 \mathrm{~K}$ and 750 - 1800 Torr. Our measured rate coefficients did not show a significant 277 pressure effect which shows that the measured data are close to the high-pressure limit. Our measured 278 CO yield was found to be $\sim$ unity at high temperatures, suggesting that the reaction R2 is the major 279 channel. We found that reaction $\mathrm{R} 1$, which also eventually produces $\mathrm{CO}$ and $\mathrm{C}_{2} \mathrm{H}_{4}$, contributes 280 negligibly small to the CO yield. Our master equation results are consistent with the experimental rate 281 constants and product yields reported in the present work. These predict that reaction R2 contributes $282 \geq 95 \%$ to cyclopentanone decomposition under our experimental conditions, whereas reaction R3 283 contributes $\lesssim 3 \%$. Our experimental results are superior to previous experiments which were probably 
284 affected by secondary free radical chain reactions. In addition, master equation calculations and 285 consequent simulation and modeling underpin an enhanced understanding of the underlying chemistry.

\section{Acknowledgments}

288 Research reported in this work was funded by King Abdullah University of Science and Technology 289 (KAUST). JRB thanks the University of Michigan for partial support of this work.

\section{List of Figures (in the Manuscript):}

297 Fig. 1. G3B3 energies [15] for the major decomposition pathways of cyclopentanone (CPO).

298 Fig. 2. Measured $[\mathrm{CO}] /[\mathrm{CPO}]_{0}$ time history for the thermal unimolecular decomposition of 299 cyclopentanone (CPO). The red line shows that CO yield reaches unity at high temperatures and/or 300 long reaction times.

301 Fig. 3. Representative absorbance time-profiles for the formation of $\mathrm{CO}$ from the thermal 302 decomposition of cyclopentanone. Black lines are the experimental traces and red lines indicate the 303 best fit of kinetic simulations at the specified temperatures. Further details of the experimental 304 conditions are provided in Table S1 of the Supporting Information.

305 Fig. 4. Arrhenius plot for the experimental and theoretical rate coefficients for the thermal 306 decomposition of cyclopentanone. Symbols: experimental data from this work; ( $\mathbf{\Delta}) 735$ - 975 Torr, 
307 ( $\mathbf{\Delta}$ ) 1050 - 1350 Torr, ( $\mathbf{a}$ )1425 - 1800 Torr. Solid lines: results of our master equation calculations for $308 \mathrm{p}=\infty, 1500$ and 750 Torr; (...) Zaras et al. [15] values for the total high-pressure limiting rate 309 coefficients. The error bars $( \pm 25 \%)$ of our measured rate constants account uncertainties in the 310 temperature $( \pm 1 \%)$ of reflected shock wave, location of time zero $( \pm 0.5 \mu \mathrm{s})$, mixture composition 311 ( $\pm 5 \%)$, CO absorption cross section $(\sim 3 \%)$. 


\section{List of Supplementary Materials:}

315 Table S1. Measured rate coefficients with the experimental conditions. The uncertainty associated with 316 the rate coefficients is $\sim \pm 25 \%$.

317 Table S2. Calculated G3B3 unscaled harmonic vibrational wavenumbers and rotational constants for 318 cyclopentenol $\rightarrow$ cyclopentadienol $+\mathrm{H}_{2}$ reaction in the potential energy surface of cyclopentanone 319 decomposition - see Zaras et al. [15] for the reaction pathways. For all other primary decomposition 320 pathways of cyclopentanone, the molecular parameters were taken from Zaras et al. [15]. Imaginary 321 wavenumber is denoted by $\mathrm{i}$.

322 Table S3. Calculated B3LYP/6-31G(d) geometries in Cartesian coordinates of the stationary points for 323 the decomposition pathway cyclopentenol $\rightarrow$ cyclopentadienol $+\mathrm{H}_{2}$.

324 Table S4. Total unimolecular rate constant $\left(\mathrm{k}_{\mathrm{uni}} / \mathrm{s}^{-1}\right)$ and statistical errors.

325 Table S5. Percent yield of $\mathrm{CO}+2 \mathrm{C}_{2} \mathrm{H}_{4}$.

326 Table S6. Percent yield of cyclopentenol $\left(\mathrm{C}_{5} \mathrm{H}_{7} \mathrm{OH}\right)$ from enolization reaction.

327 Input Data Files for The MULTIWELL Program Suite. 


\section{References}

330 [1] R. Luque, C.S.K. Lin, K. Wilson, J. Clark, Handbook of biofuels production Processes and Technologies, Woodhead Publishing, Amsterdam u.a, 2016.

[2] G.A. Strobel, B. Knighton, K. Kluck, Y. Ren, T. Livinghouse, M. Griffin, D. Spakowicz, J. Sears, Microbiology 154 (2008) 3319.

334 [3] A. Yousuf, F. Sannino, V. Addorisio, D. Pirozzi, J. Agric. Food. Chem. 58 (2010) 8630.

335 [4] X.-f. Chen, C. Huang, L. Xiong, X.-d. Chen, Y. Chen, L.-1. Ma, Bioresour. Technol. 118 (2012) $336 \quad 594$

337 [5] M. Hronec, K. Fulajtarová, Catal. Commun. 24 (2012) 100.

338 [6] A. Demirbas, Fuel Process. Technol. 88 (2007) 591.

339 [7] A. Ballistreri, D. Garozzo, M. Giuffrida, G. Montaudo, Macromolecules 20 (1987) 2991.

340 [8] J. Yang, N. Li, G. Li, W. Wang, A. Wang, X. Wang, Y. Cong, T. Zhang, Chem. Commun. 50 (2014) 3412572.

342 [9] W. Wang, N. Li, G. Li, S. Li, W. Wang, A. Wang, Y. Cong, X. Wang, T. Zhang, ACS Sustainable 343 Chemistry \& Engineering 5 (2017) 1812.

[10] H.S. Chung, C.S.H. Chen, R.A. Kremer, J.R. Boulton, G.W. Burdette, Energy \& Fuels 13 (1999) 641.

[11] O.D. Saltmarsh, R.G.W. Norrish, Journal of the Chemical Society (Resumed) (1935) 455.

[12] S.W. Benson, G.B. Kistiakowsky, Journal of the American Chemical Society 64 (1942) 80.

[13] E.R. Johnson, W.D. Walters, Journal of the American Chemical Society 76 (1954) 6266.

[14] F.M. Delles, L.T. Dodd, L.F. Lowden, F.J. Romano, L.G. Daignault, Journal of the American Chemical Society 91 (1969) 7645.

353

A.M. Zaras, S. Thion, P. Dagaut, International Journal of Chemical Kinetics 47 (2015) 439.

[16] S. Thion, C. Togbé, G. Dayma, Z. Serinyel, P. Dagaut, Energy \& Fuels 31 (2017) 2144.

354

355

A.M. Scheer, O. Welz, S.S. Vasu, D.L. Osborn, C.A. Taatjes, Physical Chemistry Chemical Physics 17 (2015) 12124.

356

[18] J. Badra, F. Khaled, B.R. Giri, A. Farooq, Physical Chemistry Chemical Physics 17 (2015) 2421.

[19] J. Bradley, Methuen, London (1962).

358

[20] M.F. Campbell, D.R. Haylett, D.F. Davidson, R.K. Hanson, Shock Waves (2015) 1.

359

W. Ren, A. Farooq, D.F. Davidson, R.K. Hanson, Appl. Phys. B 107 (2012) 849.

360

[22] L.S. Rothman, I.E. Gordon, Y. Babikov, A. Barbe, D. Chris Benner, P.F. Bernath, M. Birk, L. Bizzocchi, V. Boudon, L.R. Brown, A. Campargue, K. Chance, E.A. Cohen, L.H. Coudert, V.M. Devi, B.J. Drouin, A. Fayt, J.M. Flaud, R.R. Gamache, J.J. Harrison, J.M. Hartmann, C. Hill, J.T. Hodges, D. Jacquemart, A. Jolly, J. Lamouroux, R.J. Le Roy, G. Li, D.A. Long, O.M. Lyulin, C.J. Mackie, S.T. Massie, S. Mikhailenko, H.S.P. Müller, O.V. Naumenko, A.V. Nikitin, J. Orphal, V. 364 Perevalov, A. Perrin, E.R. Polovtseva, C. Richard, M.A.H. Smith, E. Starikova, K. Sung, S. Tashkun, J. Tennyson, G.C. Toon, V.G. Tyuterev, G. Wagner, J. Quant. Spectrosc. Radiat. 
Transfer 130 (2013) 4.

367 [23] A.M. Zaras, P. Dagaut, Z. Serinyel, The Journal of Physical Chemistry A 119 (2015) 7138.

368 [24] T.L.N. J. R. Barker, J. F. Stanton, C. Aieta, M. Ceotto, F. Gabas, T. J. D. Kumar, C. G. L. Li, L. 369 L. Lohr, A. Maranzana, N. F. Ortiz, J. M. Preses, J. M. Simmie, J. A. Sonk, and P. J. Stimac, $370 \quad$ MultiWell-2017 Software Suite, 2017.

371 [25] J.R. Barker, International Journal of Chemical Kinetics 33 (2001) 232.

372 [26] J.R. Barker, International Journal of Chemical Kinetics 41 (2009) 748.

373 [27] S.C. Smith, R.G. Gilbert, International Journal of Chemical Kinetics 20 (1988) 979.

374 [28] J.A. Miller, S.J. Klippenstein, The Journal of Physical Chemistry A 110 (2006) 10528.

375 [29] M. Akbar Ali, A. Violi, The Journal of Organic Chemistry 78 (2013) 5898.

376 [30] H. Hippler, J. Troe, H. Wendelken, The Journal of Chemical Physics 78 (1983) 6709.

377 [31] W. Forst, Unimolecular Reactions: A Concise Introduction, Cambridge University Press, 2003.

378 [32] R. Zellner, Berichte der Bunsengesellschaft für physikalische Chemie 81 (1977) 877.

379 [33] J.R. Barker, M. Frenklach, D.M. Golden, The Journal of Physical Chemistry A 119 (2015) 7451. 380 